\title{
Valores e criatividade em trabalhadores portugueses
}

\author{
Portuguese workers values and creativity
}

\author{
Leonor ALMEIDA \\ Sara IBÉRICO NOGUEIRA² \\ Adelaide Lopes JESUS ${ }^{3}$ \\ Teresa MIMOSO${ }^{3}$
}

\begin{abstract}
Resumo
Pretendeu-se caracterizar o sistema de valores e os níveis de criatividade de uma amostra de trabalhadores portugueses, bem como analisar as diferenças entre grupos com base nas variáveis: sexo, habilitações literárias, tipo de empresa e estatuto socioprofissional. O estudo da correlação entre os níveis de criatividade e os valores de vida foi, também, objeto de análise. A amostra foi constituída por 198 indivíduos, 91 do sexo masculino e 107 do sexo feminino. Para operacionalização das variáveis foram utilizados o Inventário dos Valores de Vida e o Test for Creative Thinking-Drawing Production. Entre os principais resultados salienta-se a correlação positiva entre o valor Criatividade e o nível de criatividade. Quando consideradas as variáveis sexo e empresa pública versus privada, foram encontradas diferenças estatísticamente significativas para o nível dos valores. As implicações dos resultados serão discutidas.
\end{abstract}

Unitermos: Criatividade no trabalho; Trabalhadores; Valores sociais.

\begin{abstract}
The aim of this study was to characterize the values system and the creativity levels from a sample of Portuguese workers, as well as to analyze the differences between groups based on gender, qualifications, type of business, and socio-professional status. The study of the correlation between levels of creativity and values was also examined. The sample consisted of 198 individuals, 91 males and 107 females. For operationalization of the variables the Life Values Inventory, and the Test for Creative Thinking - Drawing Production were used. Among the main results, the positive correlation between creativity level and creativity value was highlighted. When considering variables such as gender and public versus private company, statistically significant differences were found in terms of values. The implications of these results are discussed.
\end{abstract}

Uniterms: Creativity in work; Workers; Social values.

Desde a segunda metade do século XX, por força dos avanços tecnológicos, tem-se assistido a profundas transformações nas sociedades industrializadas, cujo impacto ocorre em vários níveis: econômico, social, individual e político. Atualmente, é exigido dos profissionais não apenas conhecimento técnico, mas também competências comportamentais adaptadas ao nível do trabalho de equipe, proatividade, criatividade

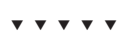

1 Instituto Superior de Gestão, Curso de Gestão do Potencial Humano, Departamento de Mestrados. R. Vitorino Nemésio, 5, 1750-306, Lisboa, Portugal. Correspondência para/Correspondence to: L. ALMEIDA. E-mail:<leonorsalmeida@gmail.com>.

2 Universidade Lusófona de Humanidades e Tecnologias. Faculdade de Psicologia, Curso de Psicologia. Lisboa, Portugal.

3 Instituto Superior de Gestão. Departamento de Mestrados, Curso de Gestão do Potencial Humano. Lisboa, Portugal. 
no desempenho, boa energia para o trabalho e flexibilidade (Mumford \& Simonton, 1997). Este aumento de exigências impulsiona os profissionais a constantes necessidades de desenvolvimento individual e profissional em que a tomada de decisão é permanente, podendo gerar situações relevantes de desgaste ocupacional e emocional, influenciando o equilíbrio holístico dos indivíduos (Basadur, 1997).

Por outro lado, também o aumento da competitividade entre as empresas, a incerteza e ambiguidade dos fatores externos exigem das organizações maior flexibilidade e capacidade de resposta, diferenciando-se, desta forma, das demais.

Reconhece-se, como forma de obter valor diferencial, a importância da produção de novas ideias, criadoras de valor para a organização, implementadas por meio do potencial criativo dos indivíduos que integram as organizações.

Pensando-se no indivíduo, os valores e as necessidades cognitivas que orientam o comportamento influenciam o grau de criatividade alcançada na medida em que, caso ele, na assunção do seu papel (ou dos seus papéis), tenha a oportunidade de satisfazer os seus valores prioritários, obtém não apenas níveis mais elevados de satisfação, mas se sente adicionalmente motivado para elaborar objetivos, metas e planos de ação para a sua concretização, tomando decisões que requerem graus diferentes de criatividade. A este propósito, as políticas, estratégias, tarefas, tecnologias, recursos e toda a equipe definem o clima organizacional e, de acordo com Ekvall e Ryhammar (1999), os produtos criativos podem-se fazer esperar se o trabalho for efetivamente significativo para os indivíduos, se estes puderem ter oportunidade de ter iniciativa e discutir abertamente, se forem estimulados e reforçados por terem novas ideias, se o risco associado às decisões puder ser tolerado e se sentirem que podem ter confiança nos seus líderes. O valor da organização em promover as novas ideias deve-se traduzir no ato de comunicação formal aos trabalhadores, bem como no reforço dessas ideias por meio de prêmios.

É, pois, nesta área da criatividade enquanto força geradora de valor e de diferenciação positiva para a empresa e para a satisfação dos trabalhadores em contexto de trabalho e de vida, obtida pela concretização

426 dos valores individuais no desempenho do papel de trabalhador, que surgiu o interesse em desenvolver este estudo.

A pesquisa aqui descrita visa a contribuir para uma melhor compreensão dos indivíduos inseridos em contexto de trabalho, objetivando-se, nomeadamente, um ajustamento mais adequado entre objetivos individuais e organizacionais e, consequentemente, maior produtividade e satisfação individuais.

Para o estudo dos valores adotou-se o modelo teórico de Brown e Crace, Holistic Value - basead Theory of Life Role Choice and Satisfaction (Brown, 1996; Brown \& Crace, 1995) e, para o estudo da criatividade, o Modelo Componencial de Urban e Jellen (1986).

O modelo teórico para o estudo dos valores de Brown e Crace identificado é o suporte teórico do teste Life Values Inventory (LVI) e serviu de base à adaptação do teste para a população portuguesa por Almeida (2007). Integra diferentes abordagens da aplicação dos valores em vários contextos da vida e, dada a sua interdependência, acentua a importância de uma perspectiva holística no desenvolvimento pessoal e de carreira dos indivíduos.

A definição de valor parte da perspectiva de Rokeach (1973), considerando os valores como a matriz referencial que guia o nosso comportamento e a avaliação do comportamento dos outros, sendo relativamente estável e permitindo-nos julgar o quão adequado esse comportamento é no momento presente, e ainda definir metas e objetivos futuros para cada uma dos contextos de vida (Brown, 1996), tendo em conta as nossas avaliações subjetivas.

O modelo teórico de Brown (1996) integra alguns aspectos da teoria de Super (1990), nomeadamente ao nível do processo de tomada de decisão e da satisfação obtida relativa ao desempenho de papéis na vida. Segundo esses autores, o indivíduo desenvolve um conjunto pequeno de valores estruturais prioritários que ele hierarquiza em um sistema axiológico e que influenciam os processos de tomada de decisão, bem como interagem em todos os papéis por ele desempenhados na vida.

Para além dos valores prioritários, são também determinantes, na tomada de decisão, o conceito de autoeficácia (Bandura, 1993), a valência que se atribui ao resultado que se pensa alcançar (Janis \& Mannn, 
1977; Vroom, 1964) e as situações disposicionais (Fiather, 1992) do indivíduo no momento da tomada de decisão.

De acordo com Brown (1996) e Super (1980; 1990), a satisfação depende da realização de vários papéis na vida, os quais, por sua vez, devem permitir satisfazer todos os valores essenciais. A importância e saliência de um papel são tanto mais evidentes quanto mais esse papel se assuma como uma oportunidade que permite satisfazer os valores principais. Assim, quando os indivíduos desempenham papéis congruentes com os seus valores e para os quais têm aptidões, tomam decisões, estabelecem objetivos e estratégias para os atingir, mostram-se motivados e desenvolvem planos de ação para responder a novos desafios.

Um dos papéis com maior relevância na vida dos indivíduos é aquele que se assume no contexto do trabalho, acabando por influenciar todos os outros, pelo que, de acordo com Brown e Crace (1995), qualquer tomada de decisão de carreira deve ser considerada atendendo ao seu impacto nos outros papéis de vida.

De acordo com Almeida (2007), o LVI permite ajudar os indivíduos a 1) cristalizar e priorizar os valores; 2) identificar os valores que esperam que venham a ser satisfeitos nas suas carreiras e nos outros papéis de vida; 3) determinar as fontes de conflitos de valores intrapessoais; 4) identificar o locus de conflitos intrapapel e, 5) identificar a fonte de conflitos interpapel.

Alguns estudos (Bridges, 1989; deVaus \& McAllister, 1991) sugerem não haver diferenças estatisticamente significativas entre o sexo masculino e feminino no que se refere a valores no trabalho. Bridges (1989) no seu estudo empírico com uma amostra de estudantes de Psicologia, encontrou diferenças, embora não estatisticamente significativas, nos valores Preocupação com os Outros, Independência e Humildade.

Erez, Borochov e Mannheim (1989) também encontraram diferenças entre dois dos oito valores usados, Independência e Privacidade, com resultado mais favorável para as mulheres. Pryor (1979) refere que, enquanto os homens tendem a valorizar mais o aspecto econômico, a independência, a segurança e objetivos a longo prazo, as mulheres tendem a valorizar as relações interpessoais, o ambiente e são mais orientadas para o curto prazo.

Os resultados de Almeida e Lopes (2004), em um estudo realizado com o Inventário dos Valores de
Vida (LVI, Life Values Inventory) em adultos trabalhadores portugueses, mostram que os mesmos apresentam uma construção de valores semelhante à obtida para a amostra americana original, embora apresentem ligeiras diferenças, principalmente na construção cognitiva dos valores Prosperidade Econômica e Pertença ao Grupo. Foram, ainda, contrastados os resultados obtidos na amostra de trabalhadores de organizações privadas com os das organizações públicas. Os resultados evidenciam a existência de diferenças estatisticamente significativas para os valores Preocupação com o Ambiente, Preocupação com os Outros, Prosperidade Econômica, Saúde e Actividade Física, Privacidade, Compreensão Científica e Espiritualidade, não obstante as limitações existentes ao nível das diferenças entre as amostras. Concretamente, os trabalhadores do setor público apresentaram índices significativamente mais elevados do que os trabalhadores do privado para os valores Compreensão Científica, Preocupação com os Outros e Preocupação com o Ambiente. Os trabalhadores do setor privado apresentaram índices significativamente mais elevados para os valores Prosperidade Econômica, Privacidade, Saúde e Actividade Física e Espiritualidade.

O outro modelo teórico adotado neste estudo é o Modelo Componencial da Criatividade, que suporta o Test for Creative Thinking-Drawing Production (TCT-DP), desenvolvido por Urban (2004). Este modelo apresenta seis componentes que funcionam em interação no processo criativo. Os primeiros três representam os componentes cognitivos: pensamento divergente e ação; conhecimento geral e pensamento de base; conhecimento de base específico e aptidões em áreas específicas. Os outros três dizem respeito aos componentes de personalidade: concentração e empenho na tarefa; motivação e motivos; abertura e tolerância à ambiguidade. Cada componente deste modelo apresenta ainda subcomponentes, não sendo nenhum deles o responsável individual pelo processo criativo, tornando-se, por isso, necessário haver uma interação de todos eles, funcionando, assim, de forma holística. Este modelo componencial obriga a uma leitura mais compreensiva do fenômeno da criatividade e pressupõe, ainda, que, para além das variáveis individuais, como a motivação e traços cognitivos, dever-se-à ter em conta a influência dos recursos materiais e dos obstáculos sociais. Assim, à avaliação das características compo- 
nenciais e de procedimento deveríamos acrescentar uma análise do produto criativo final (Urban, 1991).

Na literatura em geral não existe uma definição unânime de criatividade. Todavia, em contexto organizacional, a criatividade requerida tem que se revelar útil para a organização e envolve, entre outras variáveis, pensamento divergente e motivação intrínseca. Amabile (1983) define criatividade como a geração de ideias novas e úteis. As pessoas criativas são capazes de gerar ideias que podem dar origem a resultados ou processos inovadores que permitem uma vantagem competitiva às organizações (Amabile, Hadley \& Kramer, 2002). De acordo com Amabile (1998), Sternberg e Lubart (1996) e Csikszentmihaly (1988), o processo criativo é resultado da convergência de fatores individuais, contextuais e históricos que estão em interação e influenciam o desempenho criativo do indivíduo, nomeadamente em contexto de trabalho.

Mumford e Simonton (1997) identificaram algumas das variáveis culturais e de clima que influenciam o esforço criativo dos indivíduos e que são cruciais para o seu desenvolvimento. Em relação às variáveis culturais, deve-se ter em conta um contexto que reconheça o valor e a necessidade de correr riscos, a abertura à mudança, fomentando a aprendizagem contínua e o trabalho de equipe. Em relação ao clima, os autores identificaram o comportamento do líder na relação com o subordinado, em que o apoio e encorajamento da gestão no desenvolvimento da autoeficácia e independência e autonomia dos indivíduos são importantes para a criatividade.

Urban (2004) revela, nos seus estudos empíricos com o TCT-DP, que as pessoas com alto potencial criativo têm maior predisposição para romper com os padrões convencionais e apresentam maior apetência para correr riscos. Nesta mesma linha de pensamento inscrevem-se os estudos realizados por Amabile (1998) sobre o desenvolvimento da motivação intrínseca.

Os estudos empíricos realizados por Mumford e Simonton (1997) referem que os supervisores que demonstravam feedback positivo e concreto, transmitindo com clareza os objetivos das tarefas, melhoravam o nível de autoeficácia individual dos subordinados e promoviam o seu potencial criativo.

O estudo realizado por Mostafa (2005) com 428 trabalhadores egípcios revelou que, quanto maior o nível de habilitações escolares dos gestores, maior o seu potencial criativo, sendo que, tendo em conta a variável sexo, os resultados alcançados eram mais favoráveis aos homens do que às mulheres. Em relação à variável idade, o estudo não revelou diferenças estatisticamente significativas. $\mathrm{O}$ estudo ainda demonstrou que a aversão ao risco condiciona a produção criativa.

No estudo de Almeida e Ibérico Nogueira (2009) também se encontraram maiores níveis de criatividade entre os trabalhadores do setor privado que eram simultaneamente os que apresentavam maiores níveis de habilitações literárias.

O estudo descrito neste artigo procurou caracterizar comparativamente os valores de vida e os níveis de criatividade de um grupo de trabalhadores portugueses dos setores público e privado, bem como estudar a correlação entre os níveis de criatividade e os valores de vida. Adicionalmente, pretendeu-se estudar as diferenças entre os grupos que diferem quanto ao sexo, habilitações literárias (com ou sem ensino superior) e estatuto profissional (trabalhador versus trabalhador-estudante), considerando as variáveis valores e criatividade.

Tendo por base a revisão da literatura, esperou-se encontrar uma correlação positiva entre os cinco valores mais elevados da amostra e o resultado global do TCT-DP, bem como um resultado mais elevado, em termos de níveis de criatividade, no grupo com habilitações literárias superiores.

\section{Método}

\section{Participantes}

A amostra, de conveniência, foi constituída por 198 indivíduos trabalhadores portugueses, sendo 118 $(59,6 \%)$ sujeitos de empresas públicas e 80 (40,4\%) de empresas privadas. A distribuição por sexo identificou 107 pessoas do sexo feminino (54,0\%) e 91 do sexo masculino (46,0\%), com idades compreendidas entre 19 e 63 anos ( $M=38$ anos; DP=10,49). As idades dos sujeitos foram agrupadas de acordo com os estágios de desenvolvimento propostos por Super (1990). No grupo etário dos 15 aos 24 anos, inseriram-se 11 sujeitos participantes (5,6\%); no grupo dos 25 aos 44 anos, 133 participantes (67,2\%); e, no último grupo etário, dos 45 aos 64 anos, 54 participantes (27,3\%). 
Relativamente às habilitações literárias, 166 $(83,8 \%)$ dos sujeitos participantes tinham habilitações acadêmicas no nível do ensino secundário e 32 (16,2\%) dos participantes possuíam formação acadêmica de ensino superior (bacharelado, licenciatura ou grau superior). Ainda, em termos de estatuto profissional, 169 $(85,4 \%)$ eram trabalhadores e 29 (14,6\%) trabalhadores estudantes.

\section{Instrumentos}

Um dos instrumentos utilizados no estudo foi o Life Values Inventory, desenvolvido por Brown e Crace (1995), na sua versão portuguesa revista por Almeida (2007), o Inventário dos Valores de Vida (LVI-R). O modelo teórico que está na base de construção do teste é o Brown's Holistic Values - Basead Theory of Life Role and Satisfaction (Brown, 1996; Brown \& Crace, 1995).

O LVI-R é composto por três partes que integram um caderno de cinco páginas. A Parte I é de natureza quantitativa e as Partes II e III, de natureza qualitativa. Neste estudo apenas foi utilizada a Parte I. Esta parte inicia-se com a definição de valores e com um exemplo de como o sujeito deverá responder aos 42 itens, utilizando, para o efeito, uma escala Likert de 1 a 5 que permitirá identificar o grau em que a crença apresentada guia o comportamento do individuo, sendo que 1 significa "quase nunca guia o meu comportamento"e 5 significa "quase sempre guia o meu comportamento". As páginas 2 e 3 contêm os 42 itens (crenças) que o sujeito deverá classificar, de acordo com a escala, de 1 a 5 . Os 42 itens do teste, submetidos à análise fatorial, permitiram apurar 14 valores que são apresentados num quadro síntese na página 5 do teste. A derivação dos resultados obtida em cada um dos valores é encontrada a partir de um processo de somas combinatórias entre as várias cotações obtidas nos 42 itens. Depois de preencher o quadro síntese, devem ser assinalados os 5 valores mais elevados.

No final do teste existe, ainda, uma folha de dados demográficos, tais como a idade, sexo, área de formação, profissão, estatuto socioeconômico e situação familiar.

Ao nível das qualidades psicométricas do teste LVI-R foram utilizados, por Almeida (2007), o alfa de Cronbach para o cálculo da consistência interna do instrumento, a análise fatorial dos itens e o teste-reteste, apresentando valores ao nível da validade e precisão bastante satisfatórios, tal como também acontece na versão original americana de Brown e Crace (1995). 0 outro instrumento utilizado neste estudo foi o Test for Creative Thinking-Drawing Production (TCT-DP), desenvolvido por Urban e Jellen (1986), que possibilita avaliar o potencial criativo global dos indivíduos.

O Test for Creative Thinking-Drawing Production permite uma análise quantitativa e qualitativa da criatividade, avaliando o conteúdo, o todo, o tipo de composição e elaboração do desenho, a predisposição para o risco e para quebrar barreiras, os afetos e o humor.

A operacionalização da variável criatividade é feita pela produção de desenhos, a partir da apresentação de seis fragmentos inacabados que visam proporcionar flexibilidade nas respostas e a emergência do potencial criativo. As seis figuras fragmentadas são um semicírculo, um ponto, um grande ângulo reto, uma linha curva, uma linha descontínua e um pequeno quadrado aberto, fora do quadrado grande de resposta, desenhados numa folha A4.

A opção por um teste figurativo permite obviar algumas das principais críticas à maioria dos testes que dificilmente podem ser considerados culture-free.

Para análise dos desenhos construídos a partir destes fragmentos, foram criados 14 critérios de avaliação, atribuindo-se uma classificação que varia entre 0 e 3 ou entre 0 e 6 pontos, consoante os critérios. A soma total destas pontuações representa a classificação final indicadora do potencial criativo do indivíduo e o intervalo da sua variação é 0-72 pontos.

O teste tem duas formas, A e B, diferindo no tipo de orientação com que os elementos se apresentam, e são aplicadas ao indivíduo uma seguida da outra. Neste estudo, apenas foi utilizada a forma $A$, na medida em que em estudos preliminares portugueses (Almeida, Ibérico Nogueira \& Urban, 2007) não se verificou uma melhoria no desempenho dos sujeitos entre a forma $A$ e a forma B. A aplicação do teste pode ser feita em grupo (que não deve exceder os 10-15 participantes por cada examinador) ou individualmente a sujeitos com idades entre os 5 e os 95 anos de idade. No início da aplicação do teste é dada uma instrução específica e a sua administração terá um tempo máximo de 15 minutos para cada uma das formas (A e B). 
Em termos de qualidades psicométricas do TCT-DP, são apontados bons índices de confiabilidade intercotadores, bem como bons índices de estabilidade temporal (confiabilidade teste-reteste), por parte de vários estudos apontados por Urban (1991).

Em termos de validade fatorial, os resultados da análise fatorial efetuada a partir da amostra alemã, para ambas as formas, A e B, revelaram uma estrutura de 6 fatores: Fator I: uso do fragmento dependente e desenhos; Fator Il: composição ou conexões gráficas e de conteúdo; Fator III: perspectiva e velocidade; Fator IV: não convencionalidade e humor (forma A); Fator V: não convencionalidade e humor (forma B); e Fator VI: novos elementos e quebra do limite independente.

Quanto à validade discriminante, é importante referir que os estudos (Urban, 1991) apontaram índices fracos ou moderados de correlação entre os valores de criatividade e os valores obtidos nas Matrizes Progressivas de Raven, para os indivíduos com idades compreendidas entre 7 e 18 anos, o que pode indiciar que os testes considerados avaliam efetivamente constructos diferentes. Contudo, para os sujeitos com características de sobredotação, não se encontrou uma correlação estatisticamente significativa entre a criatividade e a inteligência geral, o que se pode dever, em parte, à pouca variância no aspecto da inteligência.

Considerando a questão da validade convergente, e dado não existirem outros instrumentos que Ihe sejam diretamente comparáveis, os poucos estudos referidos por Urban (1991) que se socorreram do TCT-DP e de outros testes de criatividade (figurativos e verbais), avaliando sobredotados verbais entre o $70 \mathrm{e}$ $10^{\circ}$ ano de escolaridade, não revelaram, na sua maioria, correlações estatisticamente significativas, provavelmente porque os instrumentos não eram diretamente comparáveis, ou seja, as dimensões de avaliação consideradas não tinham correspondência entre si.

\section{Procedimentos}

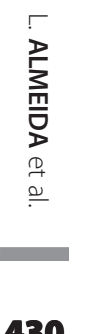

Os instrumentos LVI-R eTCT-DP foram aplicados no mês de maio, no mesmo momento e um logo após o outro, no contexto trabalho, sendo dada uma pequena explicação sobre os testes e a sua forma de preenchimento, seguindo-se um momento de esclarecimento individual, em especial em relação à realização

430 do TCT-DP.
A aplicação dos testes decorreu de acordo com as normas descritas nos manuais de cada teste, sendo estes preenchidos no momento da entrega e recolhidos pelas autoras deste trabalho, registando-se o tempo investido pelo sujeito na elaboração do TCT-DP. Depois de recolhidos todos os testes, procedeu-se à cotação dos mesmos e ao respectivo tratamento estatístico.

\section{Resultados}

Identificou-se, na amostra, os seguintes cinco valores de vida como prioritários: 1) Responsabilidade $(M=13,18 ; D P=2,17)$ - corresponde à importância dada a uma pessoa, de quem se pode depender e confiar. Consideram-se pessoas ambiciosas, responsáveis, que perdoam facilmente, são honestas, cooperativas, lógicas, autocontroladas e confiáveis; 2) Preocupação com os Outros ( $M=12,29 ; D P=1,88)$ - revelam a importância atribuída ao bem-estar dos outros. Os indivíduos que dão importância a este valor acreditam na igualdade para todos, tendem a alcançar a harmonia interna, a beleza e a paz no mundo, o perdão dos outros e a empatia. Não são os bens materiais que os movem; 3) Realização ( $M=11,97 ; D P=2,02)$ - traduzem a necessidade em aceitar novos desafios e dispõem-se a trabalhar arduamente para melhorar o seu desempenho. Regra geral, os indivíduos que revelam este valor são ambiciosos, corajosos, de confiança, com autocontrole, a sua ação é orientada por objetivos e têm apetência por atividades de risco; 4) Lealdade à Família ou Grupo $(M=11,89 ; D P=2,22)$ - os indivíduos com este valor têm necessidade de corresponder às tradições e expectativas familiares e/ou de grupo. São pessoas cuja segurança familiar assume uma especial importância. Apresentam-se como pessoas cooperativas, honestas, gentis e autocontroladas. São indivíduos que apresentam uma constante preocupação com a família, revelando, desta forma, alguma aversão ao risco; 5) Preocupação com o Ambiente $(M=11,67 ; D P=2,38)$ - diz-nos que os indivíduos com estas características consideram importante a perseveração do ambiente. São honestos, autocontrolados, preocupam-se com a beleza natural e têm facilidade em perdoar.

Os resultados mais baixos foram encontrados nos valores Humildade $(M=9,57 ; D P=1,90)$ e Saúde e Actividade Física $(M=9,76$; DP=2,26). Os índices máxi- 
mos foram encontrados em todos os valores do inventário e os mínimos em quatro valores avaliados: Prosperidade Econômica, Saúde e Actividade Física, Privacidade e Espiritualidade. É possível ver mais pormenorizadamente as médias, medianas, desviospadrão, amplitude entre valores máximos e mínimos de cada valor considerado na Tabela 1. Nesta tabela apresentam-se, ainda, os índices relativos ao teste de normalidade Kolmogorov-Smirnov, à simetria e à curtose da distribuição de cada variável (valores de vida e nível de criatividade).

Da análise da distribuição normal pelo método Kolmogorov-Smirnov, verificou-se que a grande maioria dos valores de vida, bem como a criatividade total, não seguem a distribuição normal, à exceção do valor Prosperidade Econômica, como se pode confirmar na Tabela 1. As razões para esse fato prendem-se, consoante os casos, com a assimetria ou a curtose. Contudo, atendendo ao tamanho da amostra, procedeu-se à análise de resultados com base na estatística paramétrica ou, em certos casos, na estatística não paramétrica, quando não se cumpria o critério da homogeneidade de variâncias.

Para estudar as diferenças entre grupos de trabalhadores dos setores público e privado relativamente aos valores de vida e níveis de criatividade, foi utilizado o teste $t$ de Student para amostras independentes, que permitiu concluir que não existem difrenças estatisticamente significativas entre os dois grupos quanto aos níveis de criatividade. No que diz respeito aos valores de vida, foram encontradas diferenças estatisticamente significativas nos valores Preocupação com o Ambiente e Responsabilidade, a favor dos trabalhadores do setor público (Tabela 2).

Vale salientar que esses setores (público e privado) não se encontram preferencialmente associados a nenhum nível de habilitações literárias, o que, em parte, poderia explicar as diferenças encontradas. Con-

Tabela 1

Estatística descritiva. Lisboa, Portugal, 2010

\begin{tabular}{|c|c|c|c|c|c|c|c|c|c|c|c|}
\hline \multirow[b]{2}{*}{ Critérios } & \multirow[b]{2}{*}{$\mathrm{N}$} & \multirow[b]{2}{*}{ Min. } & \multirow[b]{2}{*}{ Máx } & \multirow[b]{2}{*}{ Média } & \multirow[b]{2}{*}{ DP } & \multicolumn{2}{|c|}{ K-S } & \multicolumn{2}{|c|}{ Simetria } & \multicolumn{2}{|c|}{ Curtose } \\
\hline & & & & & & Estatística & Sig. & Estatística & $\mathrm{DP}$ & Estatística & DP \\
\hline Realização & 198 & 6 & 15 & 11,97 & 2,017 & 1,692 & 0,007 & $-0,337$ & 0,173 & $-0,150$ & 0,344 \\
\hline Pertença & 198 & 5 & 15 & 10,72 & 2,101 & 1,522 & 0,019 & $-0,142$ & 0,173 & $-0,155$ & 0,344 \\
\hline $\begin{array}{l}\text { Preocupação com } \\
\text { o ambiente }\end{array}$ & 198 & 6 & 15 & 11,67 & 2,371 & 1,628 & 0,010 & $-0,235$ & 0,173 & $-0,898$ & 0,344 \\
\hline $\begin{array}{l}\text { Preocupação com } \\
\text { os outros }\end{array}$ & 198 & 6 & 15 & 12,29 & 1872 & 1,997 & 0,001 & $-0,289$ & 0,173 & $-0,283$ & 0,344 \\
\hline Criatividade & 198 & 6 & 15 & 11,46 & 2,017 & 1,640 & 0,009 & 0,078 & 0,173 & $-0,500$ & 0,344 \\
\hline $\begin{array}{l}\text { Prosperidade } \\
\text { econômica }\end{array}$ & 198 & 3 & 15 & 10,08 & 2,505 & 1,223 & 0,100 & $-0,171$ & 0,173 & $-0,069$ & 0,344 \\
\hline $\begin{array}{l}\text { Saúde e atividade } \\
\text { física }\end{array}$ & 198 & 3 & 15 & 9,76 & 2,262 & 1,584 & 0,013 & $-0,027$ & 0,173 & 0,049 & 0,344 \\
\hline Humildade & 198 & 4 & 15 & 9,57 & 1,902 & 1,837 & 0,002 & $-0,008$ & 0,173 & 0,668 & 0,344 \\
\hline Independência & 198 & 8 & 15 & 11,64 & 1,857 & 2,072 & 0,000 & $-0,036$ & 0,173 & $-0,648$ & 0,344 \\
\hline $\begin{array}{l}\text { Lealdade } \\
\text { Família/Grupo }\end{array}$ & 198 & 4 & 15 & 11,89 & 2,223 & 1,722 & 0,005 & $-0,685$ & 0,173 & 0,605 & 0,344 \\
\hline Análise objetiva & 198 & 5 & 15 & 10,63 & 2,124 & 1,409 & 0,038 & $-0,015$ & 0,173 & $-0,154$ & 0,344 \\
\hline Privacidade & 198 & 3 & 15 & 10,91 & 2,516 & 1,684 & 0,007 & $-0,431$ & 0,173 & $-0,173$ & 0,344 \\
\hline Responsabilidade & 198 & 5 & 15 & 13,18 & 2,167 & 2,852 & 0,000 & $-1,539$ & 0,173 & 2,459 & 0,344 \\
\hline Espiritualidade & 198 & 3 & 15 & 10,13 & 3,692 & 1,407 & 0,038 & $-0,289$ & 0,173 & $-1,005$ & 0,344 \\
\hline TCT - DP & 198 & 0 & 69 & 22,72 & 12,894 & 1,954 & 0,001 & 0,788 & 0,173 & 0,172 & 0,344 \\
\hline Válidos N & 198 & & & & & & & & & & \\
\hline
\end{tabular}


tudo, o setor público está preferencialmente associado ao estatuto de trabalhador, enquanto o setor privado está preferencialmente associado ao de trabalhador-estudante $\left(\chi^{2}(1, n=198)=6,623, p=0,010\right)$. Ainda, o setor público está preferencialmente associado às profissões de administrativos e professores, enquanto o setor privado às de gestores e comerciais $\left(\chi^{2}(11, n=198)=62,92\right.$, $p=0,000)$. Relativamente às diferenças nos níveis de criatividade em função do sexo dos indivíduos, o teste t para amostra independentes não revelou quaisquer diferenças estatisticamente significativas.

No que diz respeito às diferenças de valores entre os sexos, os indivíduos do sexo feminino apresentaram valores superiores aos do sexo masculino nos valores Preocupação com o Ambiente, Preocupação com os Outros e Espiritualidade (Tabela 3).

Atendendo aos grupos etários considerados, o teste de Kruskal-Wallis não revelou diferenças estatisticamente significativas no que diz respeito os níveis de criatividade. A análise das diferenças nos valores atendendo à idade, mediante o Teste Kruskal-Wallis, evidenciou uma diferença estatisticamente significativa entre os participantes de diferentes grupos etários, no que se refere aos valores Preocupação com os Outros $\left(\chi^{2}(2, n=198)=6.97, p=0,031\right)$ e Espiritualidade $\left(\chi^{2}\right.$ $(2, n=198)=7.67, p=0,022)$, evidenciando, em qualquer um dos casos, o grupo etário mais velho (45-64) níveis mais altos.

O teste de Mann-Whitney U para a análise das diferenças nos níveis de criatividade e nos valores, atendendo às habilitações literárias, não revelou diferenças estatisticamente significativas entre os grupos com ou sem frequência de ensino superior.

O teste de Mann-Whitney $U$ para a análise das diferenças, quer na criatividade, quer nos valores, atendendo ao estatuto profissional, não revelou diferenças estatisticamente significativas entre os indivíduos trabalhadores e trabalhadores estudantes.

O estudo das correlações entre os valores de vida e os níveis de criatividade revelou uma correlação positiva, embora fraca, entre o valor Criatividade e os níveis de criatividade e uma correlação negativa, igualmente fraca, entre o valor Espiritualidade e os níveis de criatividade (Tabela 4).

Por meio do cálculo do coeficiente de determinação, verificou-se que 3,9\% da variância da criati-

Tabela 2

Análise das diferenças entre empresas públicas e privadas no que diz respeito os valores de vida. Lisboa, Portugal, 2010

\begin{tabular}{lcccccc}
\hline Valores & Tipo de Empresa & $\mathrm{N}$ & Média & Desvio-Padrão & $t$ & $\mathrm{gl}$ \\
\hline Preocupação com o Ambiente & Público & 118 & 12,07 & 2,282 & $2,908^{*}$ & 196 \\
& Privado & 80 & 11,09 & 2,393 & & 196 \\
Responsabilidade & Público & 118 & 13,73 & 1,683 & $4,19^{*}$ & 196 \\
& Privado & 80 & 12,38 & 2,533 & & \\
\hline
\end{tabular}

Nota: *Significativo $p<0,01$. N: Número de casos; gl: graus de liberdade.

\section{Tabela 3}

Análise das diferenças entre sexos relativamente a valores de vida. Lisboa, Portugal, 2010

\begin{tabular}{|c|c|c|c|c|c|c|c|}
\hline \multirow{5}{*}{ 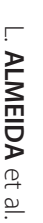 } & Valores & Sexo & N & Média & Desvio-Padrão & $t$ & $\mathrm{gl}$ \\
\hline & Preocupação com o ambiente & Feminino & 107 & 12,05 & 2,27 & $2,44^{*}$ & 196 \\
\hline & & Masculino & 91 & 11,23 & 2,42 & & \\
\hline & Preocupação com os outros & Feminino & 107 & 12,59 & 1,84 & $2,48^{*}$ & 196 \\
\hline & & Masculino & 91 & 11,93 & 1,86 & & \\
\hline & Espiritualidade & Feminino & 107 & 10,94 & 1,683 & $3,48^{* *}$ & 196 \\
\hline & & Masculino & 91 & 9,16 & 2,533 & & \\
\hline
\end{tabular}

432

Nota: ${ }^{*}$ Significativo $p<0,01$. N: Número de casos; gl: graus de liberdade. 
Tabela 4

Correlações entre valores de vida e criatividade. Lisboa, Portugal, 2010

\begin{tabular}{|c|c|c|c|c|}
\hline Valores & & Criatividade & Espirutualidade & TCT total \\
\hline \multirow[t]{3}{*}{ Criatividade } & Pearson Correlação & 1 & $0,146^{*}$ & $0,198^{* *}$ \\
\hline & Sig. (2-tailed) & & 0,040 & 0,005 \\
\hline & $\mathrm{N}$ & 198 & 198 & 198 \\
\hline \multirow[t]{3}{*}{ Espiritualidade } & Pearson Correlação & $0,146^{*}$ & 1 & $-0,157^{*}$ \\
\hline & Sig. (2-tailed) & 0,040 & & 0,027 \\
\hline & N & 198 & 198 & 198 \\
\hline \multirow[t]{3}{*}{$\mathrm{TCT}$ total } & Pearson Correlação & $0,198^{* *}$ & $-0,157^{*}$ & 1 \\
\hline & Sig. & 0,005 & 0,027 & \\
\hline & $\mathrm{N}$ & 198 & 198 & 198 \\
\hline
\end{tabular}

Nota: "Significativo $p<0,05 ;{ }^{* *}$ Significativo $p<0,01 ;$ TCT: Test of Creative Thinking; N: Número de caso.

vidade é explicada pelo valor Criatividade. Por sua vez, o valor Espiritualidade parece explicar 2,5\% da variância da criatividade.

\section{Discussão}

Uma caracterização global dos valores de vida na amostra total dos trabalhadores portugueses permitiu identificar como valores de vida prioritários os valores Responsabilidade, Preocupação com os Outros, Realização, Lealdade à Família e Preocupação com o Ambiente. É preciso salientar que esta priorização de valores já tinha sido identificada nos estudos de Almeida e Lopes (2004) com uma amostra de trabalhadores portugueses.

As diferenças estatisticamente significativas encontradas no estudo aqui descrito, no que se refere aos valores, quando comparados trabalhadores do setor público com trabalhadores do setor privado, dizem respeito aos valores Preocupação com o Ambiente e Responsabilidade, significativamente mais importantes para os trabalhadores do setor público. Considerando que se trata de uma amostra de conveniência, não é possível uma generalização dos resultados para os setores público e privado em geral. É, certamente, mais apropriado ter em conta as características estruturais e culturais das organizações em análise, bem como as características do trabalho dos colaboradores que constituem cada uma das amostras. O nível de habilitações literárias não é, para esta amostra, uma justificação a considerar, já que não foi encontrada associação alguma entre o tipo de empresa e o nível de habilitações literárias, tampouco as diferentes habi- litações literárias parecem diferenciar os sujeitos nos seus níveis de criatividade. Vale ressaltar, também, a associação entre as empresas públicas e o estatuto de trabalhador e entre as empresas públicas e as profissões de administrativos e professores. Pode-se considerar a hipótese de que os professores, devido ao seu papel de educadores, possam evidenciar uma maior Preocupação com o Ambiente e maior Responsabilidade.

Quanto à não existência de diferenças nos níveis de criatividade entre trabalhadores do setor público e do setor privado, os resultados são contraditórios com os anteriormente encontrados por Almeida e Ibérico Nogueira (2009b) que encontraram maiores níveis de criatividade nos trabalhadores do setor privado. Nesse estudo, contudo, foi encontrada uma associação entre o setor privado e o nível mais elevado de habilitações literárias, o que poderia constituir uma explicação para os resultados obtidos. As variáveis restantes - sexo, grupo etário, habilitações literárias e estatuto profissional não fizeram alterar os níveis de criatividade. Se a ausência de diferenças nos níveis de criatividade era esperada para a maioria das variáveis consideradas, atendendo a alguns estudos prévios (Almeida, Ibérico Nogueira \& Bahia, 2007), surpreendente torna-se o fato de não terem sido encontradas diferenças estatisticamente significativas entre os indivíduos com diferentes níveis de habilitações literárias, já que a maioria dos estudos das autoras tem evidenciado essa tendência a favor dos que possuem maiores habilitações literárias (Ibérico Nogueira \& Almeida, 2010). Torna-se certamente necessário atentar às profissões que têm sido desempenhadas, bem como ao número de anos na atividade profissional. 
Adicionalmente, a maior Preocupação com o Ambiente, Preocupação com os Outros e Espiritualidade, mais evidente nas mulheres do que nos homens deste estudo, vai ao encontro da maior valorização das relações interpessoais, por parte das mulheres, apontadas por vários autores. De forma semelhante, são os indivíduos mais velhos que se diferenciam dos restantes em termos dos valores Preocupação com os Outros e Espiritualidade.

Em termos organizacionais, a relação entre valores e criatividade tem constituído desde sempre um assunto de interesse, embora os estudos sobre o tema sejam escassos. Quando muito, a investigação ou se centra maioritariamente no estudo do contexto mais amplo do clima organizacional, ou, mais tangencialmente, nas medidas que especificamente avaliam o clima organizacional para a criatividade e inovação (Mathisen \& Einarsen, 2004). O estudo realizado, no que diz respeito à relação entre valores e criatividade, evidenciou que os indivíduos com maiores níveis de criatividade foram os que tiveram como um dos valores de vida prioritários a Criatividade (e.g., é importante criar coisas novas ou ter ideias novas), explicando esta uma pequena parte da variância da criatividade avaliada. Tal resultado era esperado, não tendo sido, todavia, evidenciado no estudo de Almeida e Ibérico Nogueira (2009a), que também explorou a correlação entre valores de vida e níveis de criatividade, a que provavelmente não será alheia a questão da desejabilidade social inerente a este tipo de questionários, ou a especificidade da amostra. Esses mesmos fatores podem ser igualmente responsáveis pela correlação negativa entre o nível de criatividade e o valor Espiritualidade.

Parece ser possível concluir que tanto o LVI-R quanto o TCT-DP revelaram-se instrumentos capazes de discriminar grupos de trabalhadores portugueses em relação aos seus valores de vida e níveis de criatividade. Os resultados parecem ainda evidenciar que estes se revelam bons instrumentos para discriminar os valores das pessoas e os níveis de criatividade em contexto organizacional. Tal evidência aponta para a possibilidade de utilização do LVI-R e do TCT-DP em diversas práticas organizacionais, tais como os processos de recrutamento e seleção, de gestão de carreira ou de gestão do potencial humano, principalmente no

434 que diz respeito à formação ou gestão de equipes.
Também poderão ser utilizados no desenvolvimento de equipes de trabalho de elevado rendimento.

Não obstante as vantagens que se começam a desenhar em relação à utilização do LVI e do TCT-DP em contexto organizacional, o estudo desenvolvido apresenta limitações no que se refere à caracterização, quer do contexto organizacional, quer das características do trabalho inerentes aos colaboradores que apresentam diferentes configurações de valores de vida. Ademais, separações de análise por idade e sexo poderiam ajudar a ter uma melhor percepção da situação da amostra trabalhadora; todavia, não foram realizadas neste estudo, dada a relativamente reduzida dimensão da amostra.

Neste sentido, estudos futuros deverão controlar, dentro do possível, diferenças etárias acentuadas ao nível das amostras consideradas, bem como levar em conta e avaliar de forma sistematizada aspectos organizacionais, como a cultura da organização ou mesmo as diferentes formas de gestão dos recursos humanos. Estudos futuros deverão averiguar, igualmente, qual o sentido de influência de tais características organizacionais sobre os valores e níveis de criatividade dos colaboradores que nelas trabalham. O conhecimento destes mecanismos conduzirá, certamente, a uma maior capacidade de avaliação e de intervenção ao nível da gestão das pessoas nas organizações.

\section{Referências}

Almeida, L. (2007). O inventário dos valores de vida. In M. R. Simões, C. Machado, M. M. Gonçalves \& L. Almeida (Coords.), Avaliação psicológica: instrumentos validados para a população portuguesa (pp.169-187). Coimbra: Quarteto.

Almeida, L., \& Ibérico Nogueira, S. (2009a). Creativity and values: Studies with American missionaires and Portuguese samples. Proceedings of 11 th European Congress of Psychology. Oslo, Norway, 10 July.

Almeida, L., \& Ibérico Nogueira, S. (2009b). Psychometric Evaluation of Creativity: Portuguese Studies. $11^{\text {th }}$ European Conference on Creativity and Innovations. Bruxelas, Bélgica, 28-30 Outubro.

Almeida, L., Ibérico Nogueira, S., \& Bahia, S. (2007). Assessing creativity: The Test for Creative Thinking-Drawing Production (TCT-DP) the concept, application, evaluation and portuguese studies. Proceedings of the 10th European Congress of Psychology. Prague, 12 July. 
Almeida, L., \& Ibérico Nogueira, S., \& Urban, K. (2007). Assessing creativity: The Test for Creative ThinkingDrawing Production (TCT-DP) the concept, application, evaluation and portuguese studies. Proceedings of the 10th European Congress of Psychology. Prague, 12 July.

Almeida, L., \& Lopes, M. (2004). Inventário dos valores de vida: estudos com adultos portugueses. Revista do Comportamento Organizacional e Gestão, 10(2), 189-206.

Amabile, T. M. (1998). How to kill creativity. Harvard Business Review, 76(5), 77-87.

Amabile, T. M. (1983). The social psychology of creativity. New York: Springer-Verlag.

Amabile, T. M., Hadley, C. N., \& Kramer, S. J. (2002). Creativity under the Gun. Harvard Business Review, 80(8), 52-61.

Bandura, A. (1993). Perceived self-efficacy in cognitive development and functioning. Educational Psychologist, 28(2), 117-148.

Basadur, M. (1997). Organizational development interventions for enhancing creativity in the workplace. The Journal of Creative Behavior, 31(1), 59-72.

Brown, D. (1996). A holistic, values-based model of life role decision making and satisfaction. In D. Brown \& L. Brooks and Associates. Career choice and development ( $3^{\text {rd }}$ ed., pp.337-372). San Francisco: Jossey-Bass.

Brown, D., \& Crace, R. C. (1995). Values and life role decision making. Career Development Quarterly, 44(1), 211-223.

Bridges, J. S. (1989). Sex differences in occupational values. Sex Roles, 20 (3/4), 205-211.

Csikszentmihalyi, M. (1988). Where is the evolving milieu? A response to Gruber. Creativity Research Journal, 1(1), 60-62.

de Vaus, D., \& McAllister, I. (1991). Gender and work orientation. Work and Occupations, 18(1), 72-93.

Ekvall, G. \& Ryhammar, L. (1999). The creative climate: Its determinants and effects at a Swedish University. Creativity Research Journal, 12(4), 303-310.

Erez, M., Borochov, O., \& Manheim, B. (1989). Work values of youth: Effects of sex role typing. Journal of Vocational Behavior, 34(3), 350-366.

Fiather, N.T. (1992). Values, valences, expectations, and actions. Journal of Social Issues, 48(2), 109-124.

Ibérico Nogueira, S., \& Almeida, L. (2010). Caracterização dos niveis de criatividade em adultos portugueses. Comuni- cação apresentada no $3^{\circ}$ Congresso Brasileiro: Psicologia, Ciência e Profissão. São Paulo, 3 de Setembro.

Janis, I. L., \& Mann, L. (1977). A psychological analysis of conflict, choice and commitment. New York: Free Press.

Mathisen, G. E. \& Einarsen, S. (2004). A review of instruments assessing creative and innovative environments within organizations. Creativity Research Journal, 16(1), 119-140.

Mostafa, M. (2005). Factors affecting organisational creativity and innovativeness in Egyptian business organisations: An empirical investigation. The Journal of Management Development, 24(1), 7-33.

Mumford, M. D., \& Simonton, D. K. (1997). Creativity in the workplace: people, problems, and structures. The Journal of Creative Behaviour, 31(1), 1-6.

Pryor, R (1979). In search of a concept: Work values. Vocational Guidance Quaterly, 27(1), 250-256.

Rokeach, M. (1973). The nature of human values. New York: Free Press.

Sternberg, R. J., \& Lubart, T. I. (1996). Investing in creativity. American Psychologist, 51(7), 677-688.

Super, D. E. (1990). A life-span, life-space approach to career development. In D. Brown \& L. Brooks (Eds.), Career choice and development (2a ed., pp.197-261). San Francisco: Jossey-Bass.

Super, D. E. (1980). A life-span, life-space approach to career development. Journal of Vocational Behavior, 16(3), 282-298.

Urban, K. (2004). Assessing creativity: The test for creative thinking, Drawing production (TCT-DP). The concept, application, evaluation, and international studies. Psychology Science, 46(3), 387-397.

Urban, K. (1991). On the development on creativity in children: A study with the Test of Creative ThinkingDrawing Production (TCT-DP). The Creativity Research Journal, 4(2), 177-191.

Urban, K. K., \& Jellen, H. G. (1986). Assessing creative potential via drawing production: The Test for Creative ThinkingDrawing Production (TCT-DP). In A. J. Cropley, K. K. Urban, H. Wagner \& W. Wieczerkowski (Orgs.), Giftedness: A continuing worldwide challenge (pp.163-169). New York: Trillium Press.

Vroom, V. H. (1964). Work and motivation. New York: Wiley.

Recebido em: 16/12/2011

Aprovado em: 19/12/2012 